Formación Universitaria

Vol. 6(6), 41-54 (2013)

doi: $10.4067 /$ S0718-50062013000600005

\title{
La Evaluación de Competencias Docentes en el Modelo DECA: Anclajes Teóricos
}

\author{
Rigoberto Marín ${ }^{(1)}$, Isabel Guzmán ${ }^{(1)}$, Amelia Márquez $^{(2)}$ y Manuel Peña ${ }^{(1)}$ \\ (1) Universidad Autónoma de Chihuahua, Escorza 900, Chihuahua, Chihuahua-México. \\ (e-mail: rmarin@uach.mx, iguzman@uach.mx, peachih1@yahoo.com.mx) \\ (2) Universidad Autónoma de Ciudad Juárez, Ciudad Juárez, Chihuahua, México. \\ (e-mail: ame.marquez.j@hotmail.com)
}

Recibido May. 28, 2013; Aceptado Jun. 21, 2013; Versión final recibida Ago. 23, 2013

\begin{abstract}
Resumen
En este artículo se analizan algunos anclajes teóricos del Modelo para el Desarrollo y Evaluación de Competencias Académicas. Inicialmente se revisa el concepto de competencias y sus posibilidades de trasladarlo a momentos de práctica docente y de evaluación de competencias. Específicamente, se abordan conceptos relacionados con competencias y competencias docentes. De los dos dispositivos que el modelo integra, aquí se aborda el de evaluación de competencias docentes, concebido como un momento más de su desarrollo y se proponen estrategias e instrumentos centrados en la evaluación auténtica de competencias docentes que permitan evaluar profesores en procesos de formación centrados en sus producciones. Dentro del modelo, se destaca al portafolio docente como una estrategia para la formación y evaluación de profesores y como un dispositivo de práctica reflexiva que contribuye a los procesos de formación y evaluación de competencias docentes.
\end{abstract}

Palabras clave: competencias, competencias docentes, evaluación de competencias docentes, portafolio docente.

\section{Assessment of Teaching Skills in the DECA Model: Theoretical Anchors}

\begin{abstract}
This article discusses some theoretical anchors of the Model for Development and Assessment of Academic Skills. It initially reviews the concept of skills and the possibility of going to moments of teaching practice and skill assessment. It specifically addresses concepts related to skills and teaching skills. We herein address one of the two devices included in the model: the assessment of teaching skills, conceived as one more moments of their development; strategies and tools are proposed focused on an authentic assessment of teaching skills to evaluate teachers in their training processes, focusing on their productions. Within this model, the Teaching Portfolio is stressed as a strategy to train and evaluate teachers.
\end{abstract}

Keywords: skills, teaching skills, assessment of teaching skills, teaching portfolio. 


\section{INTRODUCCIÓN}

La evaluación ocupa hoy un lugar preponderante en la agenda de la educación basada en competencias; de ello se desprende nuestro interés por abordar a la evaluación de competencias como un desafío, a la vez que como una oportunidad. Como reto buscamos establecer la congruencia entre los elementos nocionales comunes que encierra el concepto de competencia, su expresión en prácticas educativas y su traslado a contextos de evaluación, en ese proceso enfrentamos situaciones que señalan la dificultad e incluso la imposibilidad de la evaluación de competencias. Consideramos que esta inquietud, llevada al ámbito de la evaluación de competencias docentes representa una oportunidad, sobre todo si se proponen estrategias que contribuyan a promover mejores prácticas en los programas de formación-evaluación de profesores. Algunos docentes podrán plantear que dichas propuestas pueden resultar complicadas porque les demandan mayor tiempo y esfuerzo en su aplicación o que las prácticas evaluativas que han realizado convencionalmente les dan mayor seguridad. Nuestra propuesta se encamina a ofrecer una alternativa de trabajo que no resulte compleja y que pueda ser operada de manera simple en las prácticas educativas cotidianas, a fin de facilitar su incorporación gradual al repositorio de opciones e instrumentos para la evaluación de competencias que desarrollan los profesores.

Este artículo tiene como propósito analizar algunos anclajes teóricos del Modelo para el Desarrollo y Evaluación de Competencias Académicas -M-DECA- (Marín y Guzmán, 2013). En su estructura el documento presenta, de manera general, el M-DECA, sus fundamentos, propósitos y tres de sus principales componentes. En el análisis del componente conceptual se integra la revisión de la noción de competencia y las posibilidades de buscar su expresión en momentos de práctica docente y de evaluación de competencias, de manera particular se aborda lo relacionado con competencia y competencias docentes. Desde la perspectiva de formación permanente de profesores, se presentan los componentes de formación y evaluación de competencias docentes del modelo que trabajamos articuladamente en programas de formación docente. En esta comunicación se presentan las características de los componentes de formación y evaluación, dando énfasis a este segundo componente, en donde se ofrece una propuesta que analiza, comparativamente, diversas estrategias e instrumentos que incluyen, desde las formas convencionales de evaluación, hasta aquellas centradas en la evaluación auténtica de competencias docentes; esto nos ha permitido evaluar profesores en procesos de formación centrados en sus producciones. Como parte de la revisión de nuestra propuesta se analizan sucintamente tres estrategias de evaluación auténtica susceptibles de ser empleadas para la doble función de formación y evaluación de profesores: los incidentes críticos, las realizaciones o exhibiciones y el portafolio docente (Barberá, 2005; Barberá y Martín, 2009; Arbesú y Argumedo, 2010).

\section{EI MODELO PARA EL DESARROLLO Y EVALUACIÓN DE COMPETENCAS ACADÉMICAS}

En el año de 2009, grupos de investigadores pertenecientes a seis universidades mexicanas conformamos la Red para el Desarrollo y Evaluación de Competencias Académicas (REDECA); actualmente se compone por investigadores de catorce universidades, siete de ellas son extranjeras. El primer año de la REDECA se trabajó en torno del análisis, diseño y evaluación de propuestas educativas vinculadas con procesos de evaluación de la docencia en educación superior; como producto de ese esfuerzo nuestro grupo construyó una propuesta de formación-evaluación de profesores que hemos refinado y diseminado como un Modelo para el Desarrollo y Evaluación de Competencias Académicas.

En México la evaluación de la docencia es un proceso realizado por las instituciones de educación superior con finalidades y destinos que se integran básicamente en dos vertientes informativas: la administrativa y la académica (Rueda, Luna, García y Loredo, 2011). La primera, de manera externa, alimenta a organismos evaluadores y a programas de formación de profesores; y de forma interna proporciona información para la toma de decisiones y asignación de estímulos económicos a los maestros. La segunda informa a los docentes y "En esa línea de beneficios se les brindan actividades de capacitación y actualización. Sin embargo, no tienen conexión con los resultados, se trata de una oferta general que no se relaciona con las necesidades de los profesores para que mejoren sus habilidades pedagógicas en cuanto a planeación, conducción y evaluación del aprendizaje" (Rueda et al, 2011, pp. 208-209).

Con estas dos finalidades -idealmente-, los resultados de la evaluación de profesores, como corolario de dicho proceso, deben generar programas de formación continua que den a la evaluación el sentido de "diagnosticar para mejorar" capaz de crear un vínculo que se expresa en el binomio evaluación-formación (García-Cabrero, Loredo y Carranza, 2008). De esto rescatamos dos aspectos relevantes para nuestro modelo: las posibilidades de repensar este binomio de evaluación-formación en el mejoramiento de las prácticas educativas y de los sujetos involucrados, en donde el docente vaya más allá de ser simplemente el sujeto evaluado, para convertirse en un sujeto participante en su formación y evaluación. Lo anterior 
implica trabajar procesos de formación de profesores que lleven al desarrollo y evaluación de competencias docentes, transformando el binomio evaluación-formación en el bucle formación<->evaluación (Marín y Guzmán, 2012), entendido como una expresión dinámica mediante la cual, en dicho bucle, los términos se implican mutuamente y el uno requiere del otro para existir, transformándose, en distintos momentos, de evaluación-formación, en formación-evaluación. Ardoino (2001, p. 22) haciendo referencia a Dominicé menciona "...en su tesis que trata de la formación y la evaluación, hablaba ya en ese sentido de 'un proceso dentro del proceso' las ideas de interacción, de redes, de 'bucles de retroacción'...”. Díaz Barriga y Rigo (2008, p. 121) revisan varios autores que abordan la relación formación y evaluación, quienes plantean la posible y necesaria articulación entre las dos dimensiones, pero señalan que aun tratándose de una "articulación muy relevante -e incluso obvia en apariencia- no son frecuentes los estudios que se aboquen específicamente a ella". Con esta base, consideramos que la evaluación de la docencia y la formación de profesores son dos momentos paradigmáticos concomitantes de un mismo proceso $\mathrm{y}$, al entretejer estas dos nociones inseparables, asumimos que en esa madeja es posible albergar nuestros anhelos pedagógicos caracterizados por la búsqueda de modelos y estrategias mediadores de una docencia transformada y transformadora, capaz de incidir justamente en la promoción del cambio y en la mejora sustantiva de las situaciones de la vida de las personas que formamos.

Las reflexiones anteriores y la preocupación inicial sobre cómo formar<->evaluar competencias docentes, aportaron elementos para construir el M-DECA, cuyo propósito fundamental es "el desarrollo y evaluación de competencias académicas"; aplicado de manera particular en procesos de formación de profesores. El modelo se estructura por varios componentes, de los cuales en este artículo se desarrollan solo tres.

En el primer componente del modelo se fija nuestra posición respecto del discurso de las competencias; en este componente conceptual se analiza la noción de competencia y la necesidad de establecer coherencia entre los elementos estructurales del concepto de competencia, la práctica docente y la evaluación, como una estrategia de alineación constructiva (Biggs, 2005), que vincule los procesos de formación<>evaluación. En este componente también se aborda lo relacionado con la competencia y competencias docentes, este análisis conduce a la caracterización de un perfil de docente y con ello a la identificación de las siete competencias que se pretenden desarrollar y evaluar con el modelo.

Los componentes del modelo, referidos a la formación y evaluación de competencias, representan lo vertebral de los programas de formación de profesores que hemos desarrollado. En la práctica los operamos mediante dos dispositivos: uno de formación, sustentado en la pedagogía de la integración (Roegiers, 2010) y otro de evaluación de competencias docentes; este último se nutre de diversos enfoques teóricos y propone estrategias de evaluación auténtica. Mediante estos dos dispositivos se aborda la articulación entre formación y evaluación y se concibe a la evaluación de competencias docentes como un momento más de su desarrollo. Al respecto, en el modelo se proponen estrategias e instrumentos centrados en la evaluación auténtica de competencias docentes que permitan evaluar profesores en procesos de formación centrados en sus producciones.

En su estructura el M-DECA no soslaya las estrategias orientadas a su validación basadas en la propuesta de Wang y Ellet (1982), así como los métodos y técnicas que sustentan la evaluación de las acciones formativas que emprende en los programas de formación en que se apoya (Steele, 1989 y Kirkpatrick, 1999). El procedimiento que empleamos empata los seminarios del programa de formación con las etapas de validación (adopción, adaptación, evaluación, refinamiento y diseminación) y con los niveles de evaluación de acciones formativas (aprendizaje, acción, efecto y valor). Enseguida desarrollamos tres de los componentes citados del modelo.

\section{EL COMPONENTE CONCEPTUAL: PLASTICIDAD NOCIONAL Y CIERRE SEMÁNTICO}

Los intentos que buscan precisar al concepto de competencia son múltiples y a veces diversos. En el MDECA analizamos cómo el uso de la noción de competencia "se presenta como alternativa a unos modelos formativos que, tanto en el mundo del trabajo como de la escuela son insuficientes para dar respuesta a las necesidades laborales y a los problemas que depara la vida" (Zabala y Arnau, 2008, p. 31). Diferentes autores, mediante diagramas matriciales, realizan análisis comparativos de las propuestas nocionales de competencia con la intención de ofrecer su propio concepto, entre otros, Denyer, Furnémont, Poulain y Vanloubbeeck, 2009; Zabala y Arnau, 2008; Roegiers, 2010; Guzmán y Marín, 2011, esto ha brindado a la educación la oportunidad de construir nuevos significados para el concepto de competencia.

Al respecto, ante las múltiples posturas y definiciones sobre la noción de competencia, afirmamos que hemos transitado de la polisemia a la saturación de un concepto (Guzmán y Marín, 2011), lo cual sugiere la 
necesidad de arribar a cierres semánticos que impliquen detenernos para poder avanzar; por ello, en este aspecto, más que buscar anclaje en una definición concreta de competencia, consideramos importante reflexionar sobre los elementos comunes presentes en algunos de sus conceptos actuales, que permitan clarificar la complejidad del concepto y dar coherencia al empleo del concepto en su aplicación en prácticas de formación y evaluación de competencias.

En la investigación realizada, sobre la noción de competencia, se encontró que en esa amplia canasta parecen caber todos los anhelos educativos que sugieran ser inéditos, no importa que sean viables o sean nuevas o viejas utopías (Guzmán y Marín, 2011). En nuestro análisis identificamos múltiples definiciones que tienen coincidencia en ciertos elementos que se convierten en los ejes centrales del concepto de competencia, se destacan enseguida algunos de los autores revisados más relevantes, de acuerdo con la identificación que tenemos con ellos en la fundamentación del M-DECA.

Para Roegiers "La competencia es la posibilidad, para una persona, de movilizar de manera interiorizada un conjunto integrado de recursos con vistas a resolver una familia de situaciones-problema." (Roegiers, 2010, p. 89). Esta definición señala "la posibilidad" como una capacidad que potencialmente se encuentra en la persona; enfatiza el aspecto de "recursos que se movilizan" ante una determinada situación e introduce la idea de "familias de situaciones". Establece que las situaciones deben reunir diversas características para poder ser útiles como escenarios para el desarrollo de competencias, entre otras, menciona que deben ser entendidas como "situaciones-problema" o "situaciones significativas" y que estas deberán encerrar un problema, ser complejas, significativas, interactivas, a-didácticas, abiertas, inéditas, construidas y orientadas hacia los objetivos de aprendizaje.

Denyer et al, se adhieren a lo expresado en el decreto misiones de la comunidad francesa de Bélgica que define a la competencia como "la aptitud de poner en acción un conjunto organizado de saberes, de saberhacer y de actitudes que permitan realizar cierto número de tareas" (Denyer, 2009, p. 34), asimismo, rescatan lo expresado por Jonnaert respecto de que "las competencias se enfocan a través de las acciones que plantea la persona en situación y los recursos sobre los que se apoya" (Jonnaert, Barrette, Masciotra y Yaya, 2008, p. 3), buscando responder de manera más o menos pertinente a los requerimientos de la situación.

Zabala y Arnau (2008) analizan semántica y estructuralmente distintas definiciones de competencia y toman como plataforma autores tanto del mundo laboral como del educativo. Con base en ese análisis definen competencia como "la capacidad o habilidad de efectuar tareas o hacer frente a situaciones diversas de forma eficaz en un contexto determinado y para ello es necesario movilizar actitudes, habilidades y conocimientos al mismo tiempo y de forma interrelacionada" (Zabala y Arnau, 2008, pp. 43-44). En ese sentido, el término "competencia" indica no tanto lo que una persona posee, sino el modo en que actúa en situaciones concretas para realizar tareas de forma excelente. Por este motivo, las competencias tienen implícito el elemento contextual, referido al momento de aplicar estos saberes que la persona debe movilizar.

Para Perrenoud (2007, p. 11), "el concepto de competencia representará aquí una capacidad para movilizar varios recursos cognitivos para hacer frente a un tipo de situaciones". Destaca que la movilización se da siempre en situación, de esta manera, el concepto de situación se convierte en un concepto central para la adquisición de competencias ya que "es en situación que el alumno se construye, modifica o refuta los conocimientos contextualizados y desarrolla competencias a la vez situadas" (Jonnaert et al, 2008, p. 3). Respecto de los recursos cognitivos Perrenoud menciona que van más allá de los conocimientos, habilidades y actitudes presentes en la mayoría de los autores, agrega microcompetencias, informaciones, valores, esquemas de pensamiento, motores, de percepción, de evaluación, de anticipación y decisión.

Le Boterf (2001) define la competencia como "un saber actuar, es decir, un saber integrar, movilizar, transferir un conjunto de recursos (conocimientos, saberes, aptitudes, razonamientos, etc.) en un contexto determinado, para hacer frente a los diversos problemas encontrados o para realizar una tarea", agregando que "la competencia es la secuencia de acciones que combina varios conocimientos, un esquema operativo transferible a una familia de situaciones". Como se observa, sostiene la idea de esquemas de amplios recursos que se movilizan frente a situaciones o familia de situaciones.

Entre los elementos comunes que encontramos en la discusión actual sobre el concepto de competencias sobresalen: las capacidades o aptitudes que una persona pone en juego para hacer frente, disponer, actuar, saber actuar, actuar de manera competente o para movilizar un conjunto de recursos cognitivos que involucran saberes, capacidades, microcompetencias, informaciones, valores, actitudes, esquemas de percepción, de evaluación y de razonamiento, entre otras, para resolver una situación compleja. La tabla 1 resume los términos señalados que reflejan las características cardinales de la competencia. 
Tabla 1. Elementos comunes en definiciones de competencia

\begin{tabular}{|c|c|c|c|c|c|}
\hline $\begin{array}{c}\text { La competencia } \\
\text { es... }\end{array}$ & $\begin{array}{l}\text { Capacidad o } \\
\text { aptitud }\end{array}$ & Movilizar & $\begin{array}{l}\text { Recursos } \\
\text { cognitivos }\end{array}$ & $\begin{array}{l}\text { Situaciones- } \\
\text { problema }\end{array}$ & Evaluabilidad \\
\hline Roegiers (2010) & $\begin{array}{l}\text { la posibilidad } \\
\text { para una } \\
\text { persona }\end{array}$ & $\begin{array}{l}\text { de movilizar de } \\
\text { manera } \\
\text { interiorizada }\end{array}$ & $\begin{array}{l}\text { un conjunto } \\
\text { integrado de } \\
\text { recursos }\end{array}$ & $\begin{array}{l}\text { una familia de } \\
\text { situaciones- } \\
\text { problema }\end{array}$ & $\begin{array}{l}\text { con vistas a } \\
\text { resolver }\end{array}$ \\
\hline $\begin{array}{l}\text { Denyer et al } \\
(2008)\end{array}$ & la aptitud & $\begin{array}{l}\text { de poner en } \\
\text { acción }\end{array}$ & $\begin{array}{l}\text { un conjunto } \\
\text { organizado de } \\
\text { saberes, de } \\
\text { saber-hacer y de } \\
\text { actitudes }\end{array}$ & de tareas & $\begin{array}{l}\text { que permitan } \\
\text { realizar cierto } \\
\text { número }\end{array}$ \\
\hline $\begin{array}{l}\text { Zabala y Arnau } \\
(2008)\end{array}$ & $\begin{array}{l}\text { la capacidad o } \\
\text { habilidad }\end{array}$ & $\begin{array}{l}\text { y para ello es } \\
\text { necesario } \\
\text { movilizar }\end{array}$ & $\begin{array}{l}\text { actitudes, } \\
\text { habilidades y } \\
\text { conocimientos al } \\
\text { mismo tiempo y } \\
\text { de forma } \\
\text { interrelacionada }\end{array}$ & $\begin{array}{l}\text { de efectuar } \\
\text { tareas o hacer } \\
\text { frente a } \\
\text { situaciones } \\
\text { diversas }\end{array}$ & $\begin{array}{l}\text { de forma eficaz } \\
\text { en un contexto } \\
\text { determinado }\end{array}$ \\
\hline $\begin{array}{l}\text { Perrenoud } \\
(2007)\end{array}$ & una capacidad & para movilizar & $\begin{array}{l}\text { varios recursos } \\
\text { cognitivos }\end{array}$ & $\begin{array}{l}\text { para hacer frente } \\
\text { a un tipo de } \\
\text { situaciones }\end{array}$ & \\
\hline Le Boterf (2001) & un saber actuar & $\begin{array}{l}\text { un saber } \\
\text { integrar, } \\
\text { movilizar, } \\
\text { transferir }\end{array}$ & $\begin{array}{l}\text { un conjunto de } \\
\text { recursos } \\
\text { (conocimientos, } \\
\text { saberes, } \\
\text { aptitudes, } \\
\text { razonamientos, } \\
\text { etc.) }\end{array}$ & $\begin{array}{l}\text { para hacer frente } \\
\text { a los diversos } \\
\text { problemas } \\
\text { encontrados o } \\
\text { para realizar una } \\
\text { tarea }\end{array}$ & $\begin{array}{l}\text { en un contexto } \\
\text { determinado }\end{array}$ \\
\hline
\end{tabular}

\section{COHERENCIA CONCEPTUAL Y ALINEACIÓN CONSTRUCTIVA}

En el M-DECA sostenemos que algunas veces los conceptos pueden aparecer en un documento como figuras discursivas dentro del texto, sin mayor compromiso y sin trascendencia alguna. Consideramos que los elementos conceptuales que destacamos se convierten en pilares que sostienen las estrategias para la formación y la evaluación de competencias docentes. Esto es, estos elementos cobran sentido al ser transferidos a prácticas docentes y evaluación de competencias. En cuanto a la docencia, sostenemos la necesidad de transferir los elementos comunes del concepto de competencia a los procesos de prácticas educativas, así como a la evaluación de competencias docentes (Guzmán y Marín, 2011).

Lo anterior nos habla de la coherencia que requerimos establecer en el uso del concepto de competencia con relación a su desarrollo y evaluación. Al respecto, se señala que un rasgo de las competencias, que va a determinar su adquisición y evaluación, es la posibilidad de aplicarlas en contextos reales. Partimos de que una de las formas de propiciar procesos de actuación competente es colocar al alumno ante "situaciones" en las que deba movilizar sus recursos cognitivos para resolverlas con éxito. En cuanto al proceso de evaluación de competencias también deberá partir de situaciones-problema complejas que le obliguen a intervenir para resolverlas (Perrenoud, 2007; Zabala y Arnau, 2008; Jonnaert, 2008; Roegiers, 2010).

En suma, en este análisis es necesario preguntarnos hasta qué punto el concepto de competencia se ha convertido o se puede convertir en retórica y en adorno textual de los mejores discursos y documentos académicos; o hasta dónde podemos trascender del momento de lo conceptual a expresiones de práctica educativa transformada y transformadora. Con el M-DECA consideramos que el momento actual exige llevar a buen puerto prácticas educativas comprometidas y coherentes con el concepto de competencia, que mejor expresen la búsqueda de la transformación educativa.

\section{EL COMPONENTE DE FORMACIÓN: EL DESARROLLO DE COMPETENCIAS DOCENTES}

Mencionamos que con el M-DECA se pretende desarrollar y evaluar competencias docentes, en ese sentido, está orientado y alineado con la mejora de la docencia y con la posibilidad de transitar de un modelo de docencia real o inicial, a un modelo de docencia por competencias. Para la construcción del componente de formación partimos de delinear un perfil de docente que permitiera identificar las competencias docentes como un todo coherente. Con esta idea, desde la perspectiva de una práctica educativa por competencias, definimos al docente universitario como un intelectual capaz de analizar el 
proyecto institucional y curricular y, en función de ello, realizar una transposición didáctica (Chevallard, 2009) como base para el diseño de su docencia, en el cual planifica su acción de manera crítica y creativa para ponerla en práctica de manera eficaz al enfrentar situaciones-problema de docencia concretas; asimismo, es una persona que reflexiona durante la acción para reajustarla a su práctica educativa, gestionando así la progresión de los aprendizajes de sus alumnos (Perrenoud, 2007) y reflexionando posteriormente sobre la acción para evaluar el proceso y refinarlo (Schön, 1992). En ese sentido, incorporamos la idea del docente como "el profesional que reflexiona antes, durante y después de la acción" (Perrenoud, citado por Charlier, 2005, p. 150). En cuanto a la función docente, concebimos que la "competencia docente" corresponde a la parte reglada, normativa y funcional del trabajo académico que permite al profesor desempeñarse adecuadamente en el contexto de las prácticas educativas concretas de un campo profesional, esto es, de manera competente. En el M-DECA sostenemos que la competencia docente se desarrolla al enfrentarse con los problemas que la profesión docente le plantea o bien mediante los diferentes desempeños que se dan en la diversidad y complejidad de interacciones pedagógicas cotidianas en las que participa, los cuales comúnmente designamos como competencias docentes.

Con base en la pregunta ¿cuántas y cuáles competencias docentes incluir en el M-DECA? analizamos la literatura al respecto. Encontramos que diversos autores (Cano, 2005; Zabalza, 2007; Perrenoud, 2007; García-Cabrero, Loredo, Luna y Rueda, 2008; Tejada, 2009; Cardoso, Cerecedo y Vanegas, 2013), entre otros, desde distintas perspectivas aportan su visión respecto de cuáles deben ser las competencias docentes. Apoyados en esta revisión teórica y en estudios de corte empírico (Guzmán y Marín, 2012), construimos un referencial que permitió definir las siete competencias docentes que incorporamos en el modelo, estas son: 1) Desarrolla su formación continua, 2) Realiza procesos de transposición didáctica, 3) Diseña su docencia mediante dispositivos de formación y evaluación de competencias, 4) Gestiona la progresión de la adquisición de competencias, 5) Coordina la interacción pedagógica, 6) Aplica formas de comunicación educativa adecuadas y 7) Valora el logro de competencias.

Para desarrollar estas competencias empleamos como estrategia el diseño de un programa de formación de profesores que hemos aplicado en diferentes espacios y momentos (Guzmán, Marín, Zesati y Breach, 2012; Marín, Arbesú, Guzmán y Barón, 2012). En el programa formativo se trabaja inicialmente sobre la competencia "Desarrolla su formación continua"; considerando el análisis de la práctica docente propia (Perrenoud, 2007) como una acción de práctica reflexiva o como una reflexión en la acción (Schön, 1992). En este proceso el profesor analiza su práctica docente, sitúa sus competencias reales (modelo de docencia inicial), avizora las características de una docencia por competencias y plantea la necesidad de acompañamiento en su formación. Enseguida, toma como referente el proyecto y los curricula institucionales para "realizar procesos de transposición didáctica", vinculados directamente con la competencia "diseña su docencia...", que se convierte en un eje articulador y generador de las otras competencias.

En nuestro modelo, la competencia "Gestiona la progresión de la adquisición de competencias" representa un puente que oscila entre el diseño de la docencia y las competencias "coordina la interacción pedagógica y "aplica formas de comunicación educativa adecuadas". Aquí fue trabajada concibiendo que, en el proceso formativo, esta competencia adquiere "una importancia sin precedentes y sobrepasa de largo la planificación didáctica del día a día" (Perrenoud, 2007, p. 34). Desde esta perspectiva, e incorporando la visión de planeación auténtica, puede decirse que "...la competencia del profesor es doble: se incluye en la concepción, por lo tanto, en la anticipación, el ajuste de las situaciones problema al nivel y a las posibilidades de los alumnos; también se manifiesta sobre lo importante, en el tiempo real, para guiar una improvisación didáctica y acciones de regulación" (Perrenoud, 2007, p. 37).

Como parte del programa formativo construimos un dispositivo de formación que empleamos para apoyar el desarrollo de competencias docentes. La estructura del dispositivo responde a un modelo pedagógico y a una tipología de actividades que considera lo propuesto por la pedagogía de la integración (Roegiers, 2010) y algunas orientaciones sobre el desarrollo de competencias. Con estos referentes tomamos como base la pregunta "¿cómo gestionar la progresión de los aprendizajes practicando una pedagogía de situacionesproblema" (Perrenoud, 2007, p. 35). El dispositivo lo operacionalizamos mediante secuencias de aprendizaje, con las que conformamos una "guía para el docente", como base del proceso formativo. Como producto de este proceso el profesor construye una "guía para el estudiante", cuya estructura responde al mismo modelo pedagógico y tipología vivida por él en su proceso de formación (principio de isomorfismo, descrito por Mialaret, 1982). Con esta guía el profesor trabaja en colaboración con otro profesor (díada) u otros dos (tríadas) para emprender una intervención en el aula en donde aplican su proyecto de trabajo con acompañamiento mutuo e intercambio de roles (Bekerman y Dankner, 2010 y Brockbank y McGill, 2008). 
Nuestro dispositivo de formación se sustenta en secuencias didácticas que responden a la estructura que se muestra en la figura 1.

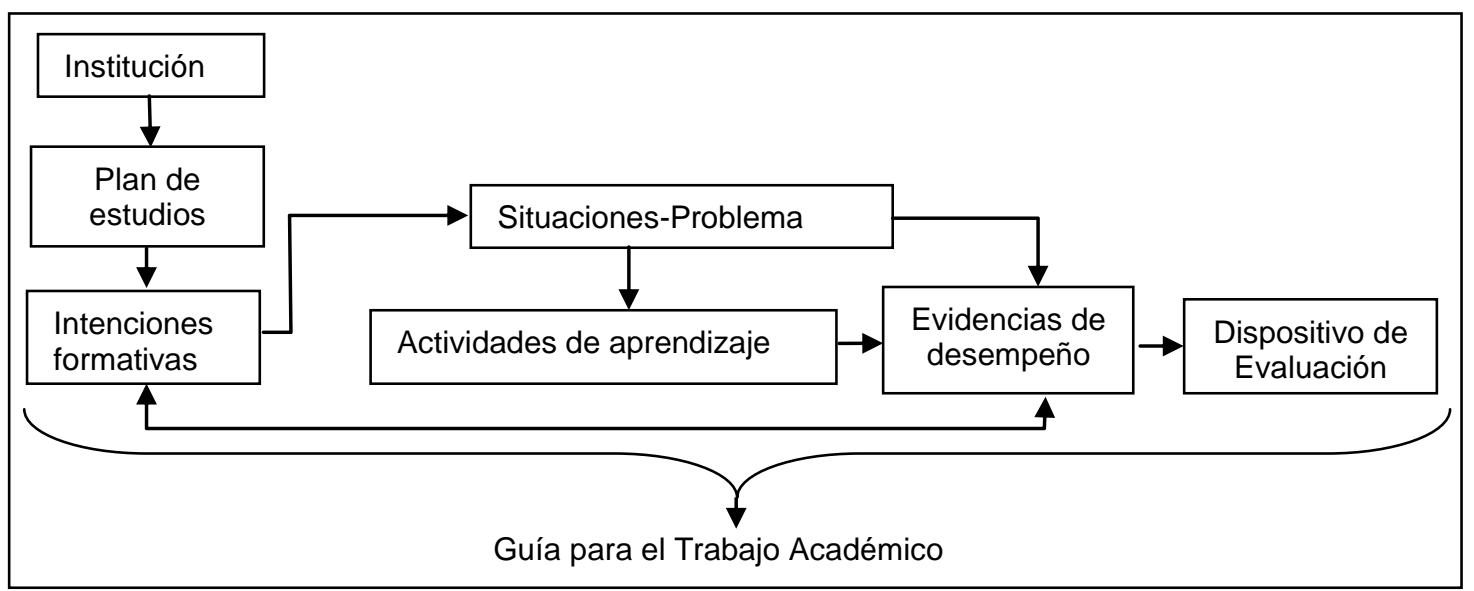

Fig. 1: Diseño de Secuencias de Aprendizaje

\section{EL COMPONENTE DE EVALUACIÓN: ESTRATEGIAS PARA EVALUAR COMPETENCIAS DOCENTES}

Para evaluar el proceso y los productos alcanzados por el profesor en nuestro proceso formativo se emplea un dispositivo de evaluación; para describirlo, partimos de diferenciar entre competencias y desempeños docentes, estimando que estos últimos permiten inferir la competencia. En ese sentido, la evaluación de competencias docentes implica valorar los desempeños del profesor al enfrentarse a situaciones-problema, partiendo de que evaluar competencias es evaluar procesos en la resolución de situaciones-problema Zabala y Arnau (2008). Con base en estos planteamientos y tratando de ser congruentes con los elementos del concepto de competencia, en el M-DECA nos preguntamos cuáles son los criterios, formas e instrumentos más adecuados para evaluar competencias docentes y si son vigentes los instrumentos empleados tradicionalmente para evaluar la docencia. Es claro que nos enfrentamos a un problema, no sólo de lo que debiera evaluarse sino, además, de cómo realizar esa evaluación, de qué manera evaluar los desempeños logrados y qué instrumentos permiten observar si efectivamente un docente es competente.

Definitivamente, la evaluación de las competencias docentes es un problema complejo; sobre todo si intentamos enfrentarlo bajo algunos de los esquemas o las formas de evaluación de la docencia convencional. En el M-DECA desarrollamos propuestas teórico-metodológicas de evaluación de competencias las cuales trascienden prácticas anacrónicas que privilegian la memorización y los saberes descontextualizados - poco motivantes y significativos para el estudiante- y con una limitada relevancia social y personal (Díaz Barriga, 2006). Si la competencia es el resultado de movilizar recursos mediante operaciones cognitivas complejas, la evaluación de la misma implicaría el poder observar esa movilización expresada en desempeños.

De conformidad con lo expuesto, en el M-DECA, nuestra propuesta se encamina a la necesidad de elegir estrategias e instrumentos pertinentes para evaluar a los profesores participantes en procesos de formación-evaluación centrados en sus producciones (Hawes, 2004). Esto considera los elementos del concepto de competencia y la discusión en torno a su evaluación, de tal forma que los instrumentos propuestos en el modelo incorporan la idea de enfrentar al docente a situaciones-problema de docencia, concretas y reales, y a la realización de tareas complejas para resolver tales situaciones. Una tarea compleja, en correspondencia, requiere del maestro una producción compleja para resolver la situación; así lo explica Bolívar: "La mejor forma de evaluar competencias es poner al sujeto ante una tarea compleja, para ver cómo consigue comprenderla y conseguir resolverla movilizando conocimientos. Los instrumentos de evaluación empleados no pueden limitarse a pruebas para ver el grado de dominio de contenidos u objetivos sino proponer unas situaciones complejas, pertenecientes a la familia de situaciones definida por la competencia, que necesitará por parte del alumno, asimismo, una producción compleja para resolver la situación, puesto que necesita conocimiento, actitudes, pensamiento metacognitivo y estratégico" (Cano, 2008, p. 12).

En ese sentido, en el M-DECA incorporamos a nuestro análisis la perspectiva de la evaluación auténtica (Herrington y Herrington, 1998; Bravo y Fernández, 2000; Rennert-Ariev, 2005; Trillo, 2005; Díaz Barriga, 2006; Palm, 2008; Monereo, 2009a), la cual se equipara con una evaluación del proceso de ejecución y con 
el realismo de la tarea que se propone (Herrington y Herrington, 1998; Monereo, 2009a). Con ello consideramos también que la evaluación de competencias es un momento más de su desarrollo. Gulikers, Bastiaens y Kirschner (2004, p. 68) lo plantean de manera categórica: "Aprendizaje y evaluación son dos caras de la misma moneda, e influyen fuertemente el uno en la otra. Para cambiar el aprendizaje del alumno en la dirección del desarrollo de competencias auténticas es necesaria una enseñanza basada en competencias auténticas, alineada con una evaluación basada también en competencias auténticas". En el M-DECA justificamos la incorporación de la evaluación auténtica de competencias docentes por la relevancia que tiene el plantear al profesor problemas semejantes o cercanos a los reales, tareas situadas y prácticas que desencadenen estrategias de resolución (Monereo, 2009a). Nuestro dispositivo de evaluación toma como base una situación-problema de docencia real a la que enfrentamos al profesor; en los programas de formación que hemos desarrollado le pedimos que "construya un "proyecto formativo" (guía para el estudiante), que contemple una docencia por competencias apegada al modelo pedagógico y tipología de actividades propuesto en el programa de formación" (competencia docente de "diseño de la docencia"). Esta situación-problema implica desempeños y ejecuciones, para ello es preciso evaluar esta competencia en su proceso y su producto.

Conscientes de que analizar la "autenticidad" de las estrategias e instrumentos empleados para la evaluación de competencias docentes requiere un lugar adicional a esta tarea, apoyados en las propuestas de Díaz Barriga, (2006) y Monereo (2009a), quienes emplean un sistema de coordenadas para situar un continuo (ejes) que considera la mayor o menor cercanía con la actividad a evaluar, construimos una propuesta (Marín y Guzmán, 2012) que presenta, para su reflexión, algunos puntos que pueden ayudar a tomar la decisión sobre qué instrumentos incorporar en un dispositivo de evaluación de competencias docentes.

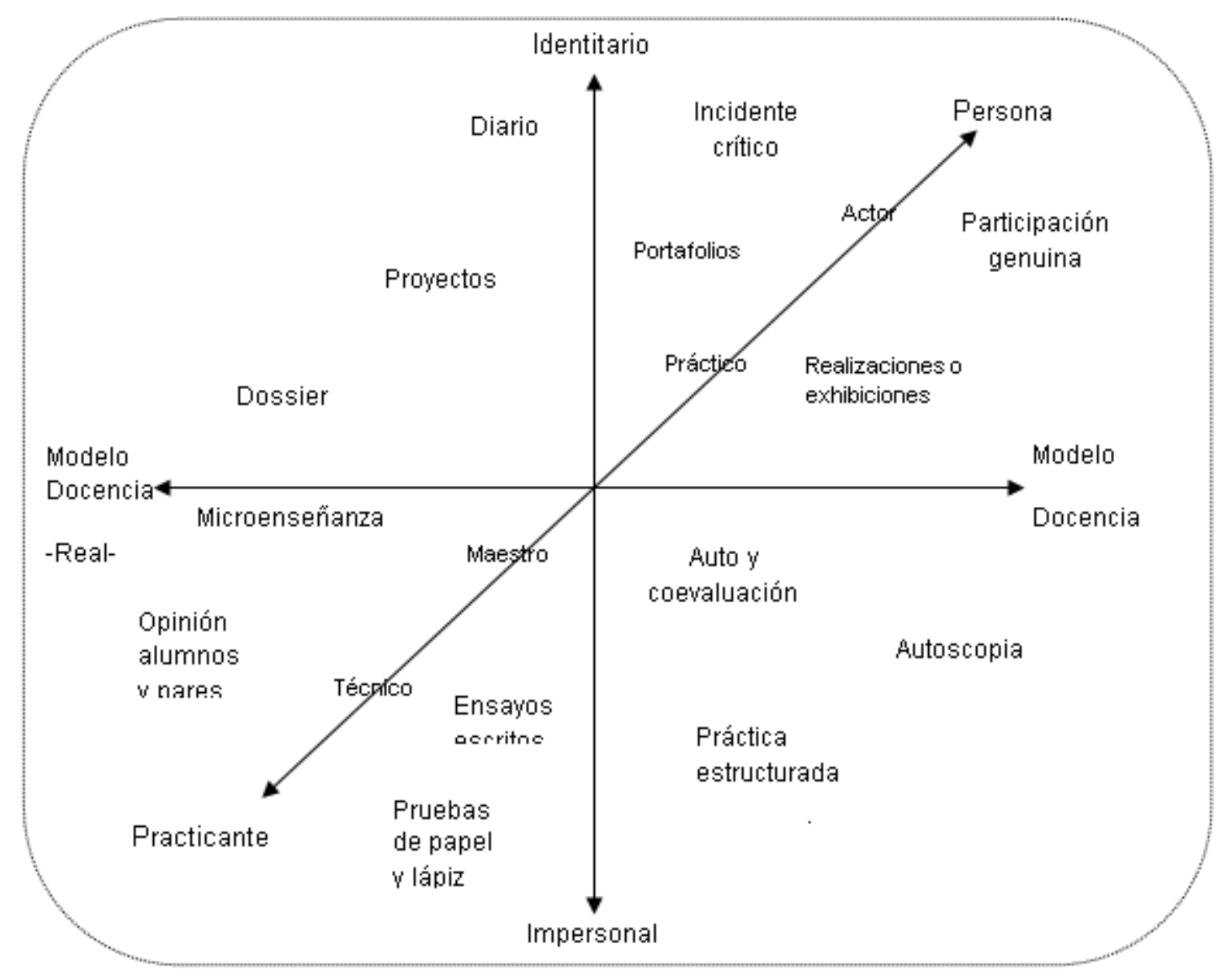

Fig. 2: Estrategias e instrumentos de evaluación de competencias docentes

En nuestra propuesta el eje de las abscisas está definido por lo que describimos como zona de desarrollo docente, sus dos polos refieren la transición de una práctica inicial (real) a una práctica por competencias. En el eje de las ordenadas colocamos lo que Monereo (2009a) designa y conceptualiza como "el nivel de enculturización identitaria (identity enculturation) que favorece". 
A estas coordenadas añadimos una dimensión que nos parece importante; está referida a los seis paradigmas relativos al oficio del maestro, propuestos como "etiquetas" mediante las cuales se identifica a un determinado tipo de maestro. De esta manera el eje de las $Z$, es un continuo en el cual se inscriben estos identificadores del profesor; de acuerdo con Paquay y Wagner (2005, p. 224) son:

1. Un "practicante artesano" que ha adquirido en el terreno ciertos esquemas de acción contextualizados.

2. Un "técnico" que ha adquirido sistemáticamente los procedimientos técnicos.

3. Un "maestro ilustrado" que domina los conocimientos.

4. Un "practicante reflexivo" que se ha construido un "saber de la experiencia" sistemático y comunicable, más o menos teorizado.

5. Un "actor social" que está comprometido en proyectos colectivos y es consciente de las apuestas antroposociales de las prácticas cotidianas.

6. Una "persona" que está en relación con los demás y en un proceso de desarrollo personal.

Considerando el plano cartesiano y sus ejes organizadores, ubicamos en sus cuadrantes algunos instrumentos que pueden ser empleados en la evaluación de competencias docentes según su grado de autenticidad (Cano, 2008).

En nuestro dispositivo hemos empleado estrategias e instrumentos de evaluación de competencias docentes que tienen que ver con los desempeños o actuaciones de los profesores, entre otros: la autoevaluación, la coevaluación (Torrez, Tena y González, 2012), la rúbrica, los proyectos, el portafolio docente, los incidentes críticos y las realizaciones o exhibiciones (Hawes, 2004). Los criterios para la inclusión de estos instrumentos, son el resultado de una investigación (Marín y Guzmán, 2012) que nos acercó a la compleja problemática de la práctica docente por competencias y a valorar el nivel de desempeño de los profesores en sus exhibiciones, quienes, de esta manera, son evaluados en sus producciones y su proceso. Dentro de la variedad de instrumentos de evaluación auténtica que hemos trabajado en nuestros programas de formación, destacamos aquí tres estrategias de práctica reflexiva que contribuyen a los procesos de formación y evaluación de competencias docentes. Mediante estas estrategias ha sido posible evaluar a profesores que han sido enfrentados a situaciones-problema específicas y reales de docencia y a la realización de tareas complejas y reflexivas como parte del proceso de resolución de tales situaciones.

Los incidentes críticos: son sucesos extraídos de la práctica cotidiana mediante observación, diálogos o entrevistas, que pueden generar dudas, sorpresa o inquietud por haberse presentado de forma inesperada, pero sobre todo porque permiten analizar situaciones que nos impactan o sorprenden, motivándonos a su reflexión. La connotación de "críticas" no implica necesariamente que presente una gravedad o riesgo extremo o fuera de control, por el contrario, regularmente se asocian al concepto de incidente crítico las ideas de sorprendente, inesperado o inquietante y útil para el profesional que analiza su propia práctica. El análisis de incidentes críticos resulta una alternativa novedosa dentro de la evaluación de competencias docentes desde la perspectiva que analizamos de formación<->evaluación (Monereo, 2009b; Marín y Guzmán, 2012).

Las realizaciones o exhibiciones: son demostraciones comprehensivas de una competencia, ya que precisan de la realización completa de una competencia. De manera general puede decirse que representan una estrategia clara e importante en la evaluación de competencias docentes. En su aplicación se estima que la persona evaluada sepa actuar o desempeñarse de manera pertinente en un contexto profesional dado. Que ante una situación-problema que se le plantee, sea capaz de seleccionar y movilizar sus recursos cognitivos a fin de resolver un problema propio de su profesión, aportando reflexiones y argumentos que dan cuenta de las razones por las que toma dichas decisiones (Hawes, 2004).

El portafolio docente: como se ha mencionado, la evaluación cobra especial significado en el ámbito de las competencias; en él se busca trascender la evaluación de los aprendizajes para avanzar hacia una evaluación más integral. Esto es, ir más allá de la obtención de evidencias que sólo reflejan la adquisición, en modo fragmentario, de conocimientos o de habilidades para avanzar hacia formas de evaluación auténtica. La evaluación de competencias proporciona la oportunidad de transformar la idea de la evaluación como control, en evaluación como mejora; y de concebirla como un momento más de aprender. La evaluación auténtica de competencias resulta relevante cuando se le plantean a la persona evaluada problemas semejantes, o cercanos, a los reales, tareas situadas (Díaz Barriga, 2006) y prácticas que desencadenen estrategias de resolución (Monereo, 2009a).

El portafolio docente se convierte en uno de los instrumentos de evaluación auténtica y de práctica reflexiva más trascendentes, pues su empleo en procesos de formación y evaluación de competencias docentes 
permite retroalimentar a las prácticas educativas. Un profesor reflexivo es, en última instancia, un profesor investigador (Cochran-Smith y Lytle, 2002; Paquay y Wagner, 2005). Este docente privilegia actividades y evidencias que selecciona e integra a su portafolio docente para su posterior análisis como parte importante de su proceso formativo. Asimismo, tiene como guía a otro compañero, quien también se concibe como un "practicante reflexivo". Finalmente, al reflexionar sobre su práctica y lo vivido, lo articula con la teoría. De esta forma, el portafolio constituye un instrumento importante para la práctica reflexiva (Burbacher, Case y Reagan, 2000). Actualmente, la formación basada en la práctica reflexiva cada vez se instaura con más fuerza como un modelo adecuado para formar profesores, superando el sistema de cursos empleados para mejorar la formación continua de los docentes. De esta manera, el portafolio docente cubre uno de sus principales propósitos al constituirse en la estrategia mediante la cual se enfatiza la práctica reflexiva (Schön, 1992) como base para la formación y el desarrollo profesional de profesores.

La importancia de incorporar el portafolio docente como un dispositivo de práctica reflexiva que contribuye a los procesos de formación y evaluación de competencias docentes se sustenta en el hecho de que permite enfrentar al docente a situaciones-problema de docencia que impliquen la realización de tareas complejas y reflexivas que conduzcan a resolver tales situaciones. El portafolio, como elemento de reflexión, representa una estrategia idónea de formación y evaluación de competencias docentes, pues a la vez que constituye una herramienta para evaluarlas, representa un detonador que desarrolla competencias complejas adicionales a las previstas en un dispositivo para la formación de profesores, convirtiéndose de esta manera en un recurso para el aprendizaje y en una estrategia de evaluación auténtica

\section{DISCUSIÓN}

EI M-DECA sustenta una propuesta para el desarrollo y evaluación de competencias que incorpora teorías, modelos pedagógicos, anhelos y aspiraciones de grandes pensadores y pedagogos; asimismo, pretende mantener congruencia teórica, pertinencia metodológica y hacer una aportación que abone a la construcción de estrategias de práctica reflexiva con momentos de acompañamiento (Schön, 1992). En el M-DECA incorporamos una perspectiva crítica de las competencias, al percibirlas, no como la panacea que va a resolver los problemas estructurales y anquilosados de los sistemas educativos actuales (Coll, 2007), sino como una estrategia para realizar prácticas incluyentes y procesos de reflexión docente.

En el recorrido de la polisemia a la saturación del concepto, más que buscar anclaje en una definición concreta de competencias, a manera de cierre semántico consideramos importante reflexionar sobre algunos elementos comunes presentes en muchos de los conceptos actuales (Guzmán y Marín, 2011), de esta manera, se parte del análisis del concepto de competencias para arribar a elementos nocionales comunes que permitan su traslado a prácticas docentes y de evaluación de competencias docentes, manteniendo de esta forma niveles de congruencia entre estos tres elementos; en este sentido, aseveramos que el concepto debe comprometer, de otra manera corre el riesgo de quedarse como un ejercicio intelectual baladí. Describimos un perfil docente que bosqueja desempeños docentes muy particulares asociados a funciones y responsabilidades propias de prácticas educativas por competencias. Estos desempeños nos acercan al concepto competencias docentes.

Una pregunta central en este artículo está referida a la evaluación de competencias como un problema complejo; considerando que en el M-DECA la evaluación cobra un nuevo significado para entenderla como un momento más del proceso de aprender. Esta visión resulta trascendente si buscamos que los profesores evalúen competencias en sus estudiantes, sobre todo si consideramos que, en las percepciones del profesorado sobre las prácticas de evaluación de los estudiantes universitarios, aún prevalecen prácticas evaluativas centradas en el aprendizaje del estudiante, siendo muy bajo el número de profesores emplean estrategias evaluadoras centradas en competencias (Cano e lon, 2012). La perspectiva de evaluación que proponemos se enriquece con momentos de práctica reflexiva y con las distintas percepciones que toman como eje a la mejora, tanto de las personas que aprenden como del contexto o ambientes de aprendizaje. La evaluación, así percibida, pretende convertirse en estrategia de formación y de desarrollo personal y profesional; esto es, se concibe a la evaluación de competencias como un espacio que se abre para seguir aprendiendo (Marín y Guzmán, 2012). Estimamos que quizá la oferta formativa hacia los profesores en servicio, pueda ser amplia, sin embargo, "todavía hay pocas experiencias formativas orientadas hacia la evaluación por competencias. Aunque el interés hacia la evaluación es cada vez mayor, todavía predomina la demanda sobre estrategias formativas y los temas «clásicos»" (Ion y Cano, 2012).

En la construcción del M-DECA se considera a la reflexión como base, encontrando en ese aspecto coincidencia con la perspectiva del portafolio docente. Al incorporar al docente a estrategias de práctica reflexiva (Brockbank y McGill, 2008) en sus espacios y tiempos de docencia, asistimos a momentos de introspección en los cuales el profesor acude a la búsqueda de explicaciones sobre su identidad profesional 
(Mérida, 2006) y, con ello, a las diversas representaciones que de ésta tiene. El portafolio docente se plantea como uno de los instrumentos sugeridos en el enfoque de la evaluación auténtica; éste incorpora en su desarrollo todos aquellos rasgos que van desde la planeación hasta la evaluación de competencias. Sin duda, dado su reciente empleo en el ámbito educativo, su incorporación se va abriendo paso en la aceptación de los docentes, llegando a ocupar un lugar importante en sus prácticas cotidianas. Sin embargo, asumimos que es necesario avanzar para darle el sentido que debe tener como un instrumento de práctica reflexiva (Lyons, 2003; Perrenoud, 2006; Medina, Jarauta e Imbernón, 2010, Imbernón y Bozu, 2012,), para, de manera progresiva, convertirlo en un verdadero "disparador" de mejores prácticas, una estrategia de formación-evaluación de profesores y una alternativa para su desarrollo profesional. Debe aclararse que el portafolio docente no está exento de críticas, sobre todo si se pretende ubicarlo como un instrumento de evaluación docente de manera rígida y lineal, o pensar en su uso como estrategia o instrumento para evaluar profesores a gran escala, pues su valor radica en sus posibilidades de estrategia de autoevaluación de prácticas reflexivas. Asimismo, si se pretende observarlo desde un punto de vista técnico, centrado en la idea de un instrumento de calificación, se corre el riesgo de desvirtuar su empleo. Otras voces consideran que es necesario dar mayor fundamento teórico al uso del portafolio como estrategia de evaluación docente, sugiriendo, además, la realización de investigaciones de corte empírico (Díaz Barriga y Pérez, 2010).

En suma, desde la visión de los procesos analizados aquí mediante el portafolio, es deseable concebirlo con el calificativo de etnográfico, pues verlo desde esta perspectiva permite "El desvelamiento y crítica emergente a los procesos de conocimiento en los que el individuo se compromete a lo largo de su ciclo vital y que, desde su protagonismo en el proceso, transforma en acción; uno recoge en su portafolio su singularidad, pero aporta al colectivo saberes; siendo intransferible, la documentación es, sin embargo, socializable" (Arraiz y Sabirón, 2007, pp. 69-70)

Si lo anterior lo enlazamos con el desarrollo y la evaluación de competencias docentes, la evaluación articulada al portafolio etnográfico integraría la autorregulación de los aprendizajes y la evaluación desde la práctica reflexiva hasta la reconstrucción personal en el desarrollo de la auténtica autonomía e independencia. Entenderlo así es asignar al portafolio etnográfico la categoría de una herramienta intersubjetiva.

\section{CONCLUSIONES}

De acuerdo a lo expuesto y discutido en este trabajo, se pueden obtener las siguientes conclusiones principales

1. EI M-DECA sustenta una propuesta para el desarrollo y evaluación de competencias que incorpora teorías, modelos pedagógicos, anhelos y aspiraciones de grandes pensadores y pedagogos.

2. EI M-DECA pretende mantener congruencia teórica, pertinencia metodológica y hacer una aportación que abone a la construcción de estrategias de práctica reflexiva con momentos de acompañamiento e incorpora una perspectiva crítica de las competencias.

3. En la construcción del M-DECA se considera a la reflexión como base, encontrando en ese aspecto coincidencia con la propuesta de estrategias e instrumentos de evaluación auténtica de competencias docentes.

4. La importancia de incorporar los incidentes críticos, las exhibiciones y el portafolio docente como estrategias de práctica reflexiva que contribuyan a los procesos de formación y evaluación de competencias docentes se sustenta en el hecho de que permite enfrentar al docente a situaciones-problema de docencia, concretas y reales, y a la realización de tareas complejas y reflexivas para resolver tales situaciones (Marín y Guzmán, 2012).

\section{REFERENCIAS}

Arbesú, M. I. y Argumedo, G., Diseño e instrumentación de portafolios para evaluar la docencia. Revista Observar (4), 28-44. (2010). Recuperado el 18 de diciembre de 2011 de http://www.odas.es/

Ardoino, J., La evaluación desgarrada: entre un balance contable y el pleno ejercicio de una función crítica plural (multirreferncial), en: Mario Rueda, Frida Díaz Barriga, y Mónica Díaz, Coordinadores, Evaluar para comprender y mejorar la docencia en la educación superior, $1^{\text {a }}$ edición, 19-35, UNAM, UAM y UABJO. México (2001). 
Arraiz, A. y Sabirón, F., El portafolio etnográfico: una herramienta facilitadora del aprendizaje a lo largo de la vida, REOP, 18(1), 65-72. (2007), recuperado el 25 de agosto de 2011 de http://www.uned.es/reop/pdfs/2007/18-1\%20\%20Ana\%20Arraiz\%20Perez.pdf

Barberá, E. y De Martín, R. E., Portafolio electrónico: aprender a evaluar el aprendizaje, UOC, Barcelona, España (2009).

Barberá, E., La evaluación de competencias complejas: la práctica del portafolios, Educere, (31), 497-503. (2005).

Bekerman, D. y Dankner, L., La pareja pedagógica en el ámbito universitario, un aporte a la didáctica colaborativa, Formación Universitaria, Vol. 3(6), 3-8 (2010), recuperado el 21 de julio de 2013 de http://www.scielo.cl/pdf/formuniv/v3n6/art02.pdf

Biggs, J., Calidad del aprendizaje universitario. 1a edición, 7-295, Narcea ediciones, Madrid, España (2005).

Bravo, A. y Fernández J., La evaluación convencional frente a los nuevos modelos de evaluación auténtica, Psicothema, 12 (2), 95-99. (2000). Recuperado el 4 de noviembre de 2009 de http://www.psicothema.com/pdf/524.pdf

Brockbank, A. y Mcgill, I., Aprendizaje reflexivo en la educación superior, 2ª edición, 7-311, Morata, Madrid, España (2008).

Burbacher, J., Case, Ch. y Reagan, T., Cómo ser un docente reflexivo, 1a edición, 9-171. Gedisa, Barcelona, España (2000).

Cano, E. e Ion, G., Prácticas evaluadoras en las universidades catalanas: hacia un modelo centrado en competencias, Estudios sobre educación, Vol, 22 (2012) Recuperado el 14 de enero de 2013 de http://dspace.unav.es/dspace/handle/10171/22627

Cano, E., Cómo mejorar las competencias de los docentes. Guía para la autoevaluación y el desarrollo de las competencias del profesorado, 3-213, Graó, Barcelona, España (2005).

Cano, E., La evaluación por competencias en la educación superior, Profesorado. Revista de currículum y formación del profesorado. (2008). Recuperado el 23 de enero de 2010 de http://www.ugr.es/local/recfpro/rev123COL1.pdf

Cardoso, E, Cerecedo, M. y Vanegas, E., Las competencias docentes en los programas de posgrado en Administración. Un Estudio de Diagnóstico, Formación Universitaria Vol. 6(2), 43-50 (2013) recuperado el 21 de julio de 2013 de http://www.scielo.cl/pdf/formuniv/v6n2/art06.pdf

Cochran-Smith, M. y Lytle, S., Dentro-fuera. Enseñantes que investigan, Akal, Madrid, España (2002).

Coll, C., Las competencias en la educación escolar: algo más que una moda y mucho menos que un remedio, Innovación Educativa, Aula de Innovación Educativa, 161, 34-39 (2007).

Charlier, E., Cómo formar maestros profesionales. Por una formación continua vinculada con la práctica, en Léopold Paquay, Marguerite Altet, Évelyne Charlier y Philippe Perrenoud (coords), La formación profesional del maestro, estrategias y competencias, 139-169, Fondo de Cultura Económica, México. D.F. (2005).

Chevallard, Y., La transposición didáctica. Del saber sabio al saber enseñado, 3ª edición, 3ํㅡㄹ reimpresión, 5-196. Aique, grupo editor. Buenos Aires, Argentina (2009).

Denyer, M., Furnemont, D., Poulain, R. y Vanloubbeeck, P., Las competencias en la educación. Un balance. 1a edición, 13-201, Fondo de Cultura Económica, México, D.F. (2009).

Díaz Barriga F. y Pérez, M. M., El portafolio docente a escrutinio: sus posibilidades y restricciones en la formación y evaluación del profesorado Observar 2010. 4, 6-27, (2010). Recuperado el 23 de marzo de 2012 de www.odas.es/site/magazine.php

Díaz Barriga, F. Enseñanza situada: Vínculo entre la escuela y la vida. 1aㅡ edición, 1-171, McGraw Hill, México, D.F., (2006).

Díaz Barriga, F. y Rigo, M. A., Posibles relaciones entre formación y evaluación de los docentes, En Mario Rueda Beltrán (Coord.), La evaluación de los profesores como recurso para mejorar su práctica, 1a edición, 121-160, IISUE-UNAM, México, D.F. (2008).

García-Cabrero, B., Loredo, J. y Carranza, G., Análisis de la práctica educativa de los docentes: pensamiento, interacción y reflexión, Revista Electrónica de Investigación Educativa, Especial, (2008). Recuperado el día 30 de octubre de 2010 de http://redie.uabc.mx/NumEsp1/contenido-garcialoredocarranza.html 
García-Cabrero, B., Loredo, J., Luna, E., y Rueda, M., Modelo de Evaluación de Competencias Docentes para la Educación Media y Superior. Revista Iberoamericana de Evaluación Educativa 2008-Volumen 1 , Número 3. (2008b) Recuperado el 30 de octubre de 2009 de http://www.rinace.net/riee/numeros/vol1-num3_e/art8.pdf

Gulikers, J., Bastiaens, T. y Kirschner, P., A five-dimensional framework for Authentic Assessment, (2004).

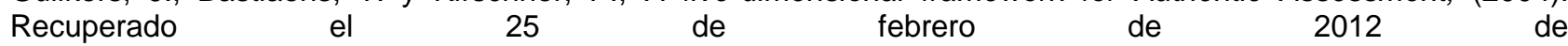
http://racetothetopvolusia.wikispaces.com/file/view/A+Five+Dimensional+Framework+for+Authentic+Assessment. pdf

Guzmán I., Marín, R., Zesati, G. y Breach, R. M., Desarrollar y evaluar competencias docentes: estrategias para una práctica reflexiva. Voces y Silencios: Revista Latinoamericana de Educación, Vol. 3, No. 1, 22-40 (2012) recuperado el 18 de enero de 2013 de http://vocesysilencios.uniandes.edu.co/index.php/vys/article/viewFile/110/291

Guzmán, I. y Marín, R., La competencia y las competencias docentes: reflexiones sobre el concepto y la evaluación, (2011). Recuperado el 25 de abril de 2011 de www.aufop.com/aufop/uploaded_files/articulos/1301588498.pdf

Guzmán, I., Marín, R., Ángeles, E., Moreno, S. A. y López, J., Identificación de competencias docentes en el oficio de enseñar: el caso de tres campos profesionales, en Edith Cisneros Chacón, Benilde García Cabrero, Edna Luna y Rigoberto Marín Uribe (coords.), Evaluación de competencias docentes en la educación superior, 1aㅡ edición, 159-202, Juan Pablos Editor, México (2012).

Hawes, G., Evaluación de logros de aprendizaje de competencias (2004). Recuperado el 18 de noviembre de 2009 de http://www.gustavohawes.com/Educacion\%20Superior/2008EvaluacionAprendizajes.pdf

Herrington, J. y Herrington, A., Authentic conditions for authentic assessment: Aligning task and assessment. (1998). Recuperado el 15 diciembre de 2009 de http://edserver2.uow.edu.au/ janh/Assessment/Authentic\%20Assessment_files/HERDSAHerringtonFinal.doc

Imbernón, F. y Bozu, Z., El portafolio docente como estrategia formativa innovadora del profesorado novel universitario, (2012). Recuperado el 10 de enero de 2012 de www.revistaeducacion.mec.es/doi/358_077.pdf

Ion, G. y Cano, E., La formación del profesorado Universitario para la implementación de la evaluación por competencias. Educación XXI. (15) 2, 249-270 (2012), recuperado el 20 de diciembre de 2012 de http://www.uned.es/educacionXX1/pdfs/15-02-11.pdf

Jonnaert, Ph., Barrette, j., Masciotra, D. y Mane Yaya, M., La competencia como organizadora de los programas de formación: hacia un desempeño competente. Profesorado. Revista de currículum y formación del profesorado, 12, 3 (2008). Recuperado el 20 de octubre de 2011 de http://www.ugr.es/local/recfpro/rev123ART3.pdf

Kirkpatrick, D., Evaluación de acciones formativas. Los cuatro niveles, 1a Edición, 1-289, Epise, Barcelona, España (1999).

Le Boterf, G., Ingeniería de las competencias, 1a edición, Ediciones Gestión 2000, Barcelona, España (2001).

Lyons, N., El portafolio y sus consecuencias: formación de profesionales reflexivos, en Nona Lyons (Comp.), El uso de portafolios. Propuestas para un nuevo profesionalismo docente, $1^{\text {a }}$ edición, 325-346, Amorrortu. Buenos Aires, Argentina (2003).

Marín, R. y Guzmán, I. Modelo para el desarrollo y evaluación de competencias académicas. Manuscrito no publicado, Universidad Autónoma de Chihuahua, Chihuahua, México (2013).

Marín, R. y Guzmán, I., Formación<->evaluación: una propuesta para el desarrollo y evaluación de competencias docentes, en E. Cisneros Chacón, B. García Cabrero, E. Luna y R. Marín Uribe (coords.), Evaluación de competencias docentes en la educación superior, 1a edición, 203-247, Juan Pablos Editor, México, D.F. (2012).

Marín, R., Arbesú, M. I., Guzmán, I. y Barón, V. El empleo del portafolio en la formación-evaluación de competencias docentes. Voces y Silencios: Revista Latinoamericana de Educación, Vol. 3, No. 1, 5-21, recuperado el 18 de enero de 2013 de http://vocesysilencios.uniandes.edu.co/index.php/vys/article/view/109/294

Medina, J. L., Jarauta, B. e Imbernón, F. La enseñanza reflexiva en la educación superior. (2010). Recuperado el 23 de diciembre de 2010 de www.octaedro.com/pdf/16517.pdf

Mérida, R. Nueva percepción de la identidad profesional del docente universitario ante la convergencia europea. (2006). Recuperado el 22 de diciembre de 2011 de http://www.redie.uabc.mx

Mialaret, G., Principios y etapas de la formación de educadores, en M. Debesse y G. Mialaret. La formación de enseñantes, 163-185, Oicos-Tao, Barcelona, España (1982). 
Monereo, C., La autenticidad de la evaluación, En Monserrat Castelló (Coord.), La evaluación auténtica en enseñanza secundaria y universitaria, 1aㅡ edición 15-32, Ed. Cast. Edebe, Barcelona, España (2009a).

Monereo, C., La formación del profesorado: una pauta para el análisis e intervención a través de incidentes críticos. (2009b). Recuperado el 22 de octubre de 2009 de http://www.rieoei.org/rie52a08.htm

Palm, T., Performance Assessment and Authentic Assessment: A Conceptual Analysis of the Literature. (2008). Recuperado el 15 diciembre de 2009 de http://pareonline.net/getvn.asp? $v=13 \& n=4$

Paquay, P. y Wagner, M. C., La formación continua y videoformación: qué habilidades se deben priorizar, en Léopold Paquay, Marguerite Altet, Évelyne Charlier, Philippe Perrenoud (Coords.), La formación profesional del maestro. Estrategias y competencias, 1a edición, 222-264, Fondo de Cultura Económica, México, D.F. (2005).

Perrenoud Ph., Desarrollar la práctica reflexiva en el oficio de enseñar, profesionalización y razón pedagógica, $2^{\text {a }}$ edición, 9-224. Graó, Barcelona, España (2006).

Perrenoud, Ph., Diez Nuevas Competencias Para Enseñar, 4ª Edición, 7-168. Graó, Barcelona, España (2007).

Rennert-Ariev, P., A theoretical model for the authentic assessment of teaching, (2005). Recuperado el 15 diciembre de 2009 de http://pareonline.net/getvn.asp? $v=10 \& n=2$

Roegiers, X., Pedagogía de la integración. Competencias e integración de los conocimientos en la enseñanza, 1 a edición, 7-386, FCE, México, D.F. (2010).

Rueda, M., Luna, E., García, B. y Loredo, J., Resultados y recomendaciones, En M. Rueda (coordinador), ¿Evaluar para controlar o mejorar? Valoración del desempeño docente en las universidades, 1ª edición, 197-222, IISUE-UNAM, México (2011)

Schön, D., La formación de profesionales reflexivos: hacia un nuevo diseño de la enseñanza y el aprendizaje en las profesiones. 1aㅡ edición, 7-310. Paidós/MEC, Madrid, España (1992)

Steele. S., The evaluation of adult and continuing education, en S. B. Merriam y P. M. Cunningham (eds.), Handbook of adult and continuing education, 261-272, Jossey-Bass, San Francisco, USA(1989).

Tejada, J., Competencias docentes, Profesorado. Revista de Curriculum y Formación del Profesorado, 13 (2), 1 15, (2009).

Torrez, H., Tena, M. y González, L., Co-evaluación de competencias en el proyecto final de carrera: aplicación a la titulación de Administración y Dirección de Empresas. Formación Universitaria. Vol. 4(5), 37-44 (2011), recuperado el 21 de diciembre de 2012 de http://www.scielo.cl/scielo.php?pid=S071850062011000500006\&script=sci_arttext

Trillo, F., Competencias docentes y evaluación auténtica: ¿falla el protagonista? (2005). Recuperado el 20 de enero de 2010 de http://www.unrc.edu.ar/unrc/academica/pdf/cuadernillo03.pdf

Wang, M. \& Ellett, Ch., Program validation: the state of the art, en Topics in Early Childhood Special Education. (1982), Recuperado el día 21 de octubre de 2010 de http://tec.sagepub.com/content/1/4/35

Zabala, A. y Arnau, L., 11 Ideas clave: como aprender y enseñar competencias, 4ª reimpresión, 7-226. Graó, Barcelona, España (2008).

Zabalza, M., Competencias docentes del profesorado universitario. Calidad y desarrollo profesional, $2^{\underline{a}}$ Edición, primera reimpresión, 7-229, Narcea, Madrid, España (2007). 